\title{
COSMETIC CONTAMINATION AWARENESS AMONG ADOLESCENT FEMALES
}

\author{
ILANKIZHAI RJ ${ }^{1 *}$, GAYATHRI R $^{2}$, VISHNUPRIYA V ${ }^{2}$
}

${ }^{1}$ Department of Biochemistry, Saveetha Dental College and Hospitals, Saveetha University, Chennai - 600077 , Tamil Nadu, India. ${ }^{2}$ Department of Biochemistry, Saveetha Dental College and Hospitals, Saveetha University, Chennai - 600 077, Tamil Nadu, India. Email: ilankizhairj@outlook.com

Received: 07 May 2016, Revised and Accepted: 14 May 2016

\section{ABSTRACT}

Objective: Cosmetics, in today's world, have become a part and parcel of every women's life. The majority of the women who use cosmetics are unaware of the fact that makeup can harbor a variety of infections if not used in the appropriate manner. The purpose of the study is to determine the awareness of cosmetic contamination among adolescent females and spread awareness regarding the different ways to prevent the harmful effects that these contaminated cosmetics can have on the health of the users.

Methods: This is a survey-based study. This study was conducted by observing cosmetic usage practices of adolescent females through the medium of analyzing questionnaires. The data collected were compiled and analyzed statistically.

Results: The responses of the participants reveal that there is a rapid increase in the awareness regarding cosmetic contamination among adolescent females. Out of the 100 adolescent females who completed the survey, $87 \%$ of the females are aware of the presence of lead, carcinogenic substances, and preservatives that can become toxic over a period of time, the rest $13 \%$ are unaware of the fact and the harmful effects these can cause if not handled properly.

Conclusion: The results of the study show the increase in cosmetic usage patterns and cosmetic contamination awareness among the adolescent females in the past few decades.

Keywords: Cosmetic contamination, Microbial, Contamination, Awareness, Adolescent females, Toxicity.

\section{INTRODUCTION}

Cosmetics - the word has become an inevitable part of every women's life.

The word "cosmetics" is derived from the Greek word Kosmetikos which means skilled at decorating. Cosmetics are also colloquially known as makeup or personal care products which are used to enhance the appearance or odor of the human body. They are generally mixtures of chemical compounds, with some derived from natural sources and some synthetic [1]

The Food and Drug Administration (FDA), which regulates cosmetics, defines cosmetics as "intended to be applied to the human body for cleansing, beautifying, promoting attractiveness, or altering the appearance without affecting the body's structure or functions." $[2,3]$. Each and every day, it is estimated that we put up to 10 different personal care products on our bodies. This includes shampoo/ conditioner, lotions, soap, toothpaste, deodorant, lip balm, sunscreen, shaving products, cosmetics, and insect repellants.

Whether it's for putting on a show or getting ready for an occasion we've all used or seen it being used at least once in our lives. Cosmetics is used by both genders but is predominantly used by more females than males at the global level. Makeup plays a vital role in altering a woman's appearance and that is the reason for its huge popularity among today's young generation. Cosmetics can do wonders for women, but if not handled properly makeup can pose a threat to their health [4].

A majority of the women who use cosmetics are unaware of the fact that makeup can harbor a variety of infections if not used in the appropriate manner.

Some women even share makeup and applicators with friends and family, increasing their chances of infection. Others do not replace makeup until it's completely finished despite how long ago they purchased it. Cosmetic contamination awareness is very poor among the users of all age groups.

\section{Cosmetic contamination}

Cosmetic contamination is an issue of great importance in today's world. FDA states that it is not necessary for cosmetic products to be sterile; however, they must not be contaminated with pathogenic micro-organisms [5]. There are numerous ways in which the cosmetic products can be contaminated. The most common way of contamination is in use contamination, with the user repeatedly applying makeup to the skin using an applicator, or finger and then putting it back into the makeup. Another possible way makeup can be contaminated is by poor handling procedures during manufacturing, which can cause defects in the preservative capacities of makeup. Storage method of makeup can also determine whether it is more likely for bacteria to grow or not [4].

\section{Microbial contamination}

Microbial contamination of cosmetics has been studied since long. Microbial contamination of cosmetic products is very crucial because of their daily use and direct contact with the skin. Cosmetic products are at high risk for microbial contamination from various sources such as environment, consumer's hands, body sweat, and during the time of manufacturing [6]. It is known that eye cosmetics have the ability to harbor numerous microorganisms. Furthermore, the unrestricted use of cosmetic testers, for which there are no sterile applicators, can spread bacteria from one person to another and possibly cause eye infections [7]. A proper use of makeup may reduce the risk of infection. Flip caps and pump tops are the best ways of closing shampoos and lotions [8]. It is even concluded that poorly preserved cosmetics can be protected with the right packaging. 
The quality of makeup is also an important factor in whether makeup can become contaminated or not. Preservative systems in cosmetics inhibit the spoilage of the products and prevent the growth of pathogens [9]. Preservative systems, however, can lose their effectiveness if stored for a longer period of time [10].

Sometimes, labels on cosmetics also lack essential information that ensures the safety of the product. In some studies, labels were found to not have dates of manufacture or dates of expiration [11]. Even so, it is still up to the consumer to properly handle makeup and applicators to avoid infection [4].

The age range of female makeup users has increased widely across the decades. The age that females begin wearing makeup gets younger with every new generation. If consumers are aware about the proper ways of handling the products, they should be able to have a low incidence of infection [4]. The purpose of this study is to determine the awareness of cosmetic contamination among adolescent females and the buying behavior of consumers.

\section{METHODS}

This is a survey based cross-sectional study. A survey method was adopted as a means of data collection because it provided the best possible way to determine the awareness of female adolescents regarding the use of cosmetics [4]

This study was conducted by observing cosmetic usage practices of adolescent females through the medium of analyzing questionnaires. The survey was basically arranged in three sections in which the first section consisted of questions regarding the age and the usage of makeup. The second section consisted of questions regarding the usage pattern of different personal care products. The third section included questions regarding the awareness and shopping pattern of the consumers. The data were collected from a sample size of hundred adolescent females (12-19) from different regions and social class. Adolescent females were chosen for this study because females are the primary users of makeup [4]. The data collected were compiled and analyzed statistically.

\section{RESULTS}

The sample size of 100 was considered for this survey based research. The questionnaires were handed over to adolescent females (12-19) across different regions and social class and 100 completed questionnaires were collected and analyzed for results.

Out of 100 people who filled the questionnaires 86 people use makeup and the other 14 do not use makeup (Table 1).

The graph below gives information regarding the usage pattern of different personal care products.

From Fig. 1, it is clearly evident that body care products which include bar soaps, body wash, moisturiser, facial lotion, body lotion, sunscreen lotion, perfume, and deodorants have been used more frequent when compared to the other personal care products. Hair care products such as shampoo and conditioner are used by most people few times a week. Nail polish and nail paint remover are more predominantly used once or twice per month unlike the other products which are used on a more frequent basis. The usage pattern lip care products and eyeliner and eye shadow does not show a prominent pattern, and the frequency of usage is more irregular among different groups of females according to their priorities and preferences.

The most used makeup applicators were bristle brushes (29\%) and fingers $(22 \%)$ and the least used were roll on $(11 \%)$ and cotton swabs $(17 \%) .21 \%$ of females use other applicators for applying makeup (Table 2).
Table 1: Percentage of females using makeup

\begin{tabular}{ll}
\hline Makeup use & Percentage (\%) \\
\hline Uses makeup & 86 \\
Do not use makeup & 14 \\
\hline
\end{tabular}

Table 2: Percentage of females using different applicators

\begin{tabular}{lll}
\hline S.N & Applicators & Percentage (\%) \\
\hline 1 & Bristle brushes & 29 \\
2 & Cotton swabs & 17 \\
3 & Roll on & 11 \\
4 & Fingers & 22 \\
5 & Others & 21 \\
\hline
\end{tabular}

Sample size-100

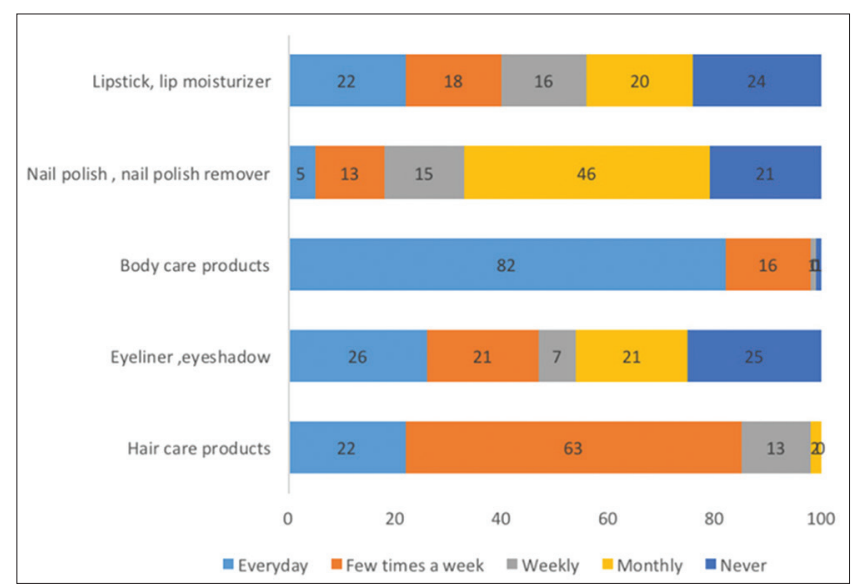

Fig. 1: Frequency of usage of different personal care products. Sample size-100

The number of females who share or borrow makeup is outlined in Fig. 2. In total, out of 100 females who participated in the study 57 do not share their makeup with anyone. Out of the remaining 43 who share their makeup nearly $70 \%$ share it with one or two members who are usually their siblings and family members. Nearly $25 \%$ of the people who share their makeup are not sure of the number of people with whom they share their cosmetics with.

Out of the 100 adolescent females who completed the survey, $52 \%$ of 41 females are aware of the expiry date and use the products only until 42 the expiry date. Whereas $28 \%$ of females have no awareness about the 43 expiry date and use it until the product finishes (Fig. 3).

Approximately, 54\% of the people who completed the survey buy cosmetic products based on their brand and 28\% take advice from friends and very few people give importance to packaging, price and advertisements (Fig. 4).

\section{Cosmetic contamination awareness}

According to the data collected, out of the 100 adolescent females who completed the survey, $87 \%$ of the females are aware of the presence of mercury, lead, carcinogenic substances, and preservatives that can become toxic over a period of time, the rest $13 \%$ are unaware of the presence of these substances in the cosmetic products and do not know the harmful effects these can cause if not handled properly.

The awareness of the users regarding the usage of cosmetic testers and the importance of the label of ingredients in the cosmetic products were also taken into consideration, and it turned out that majority of 


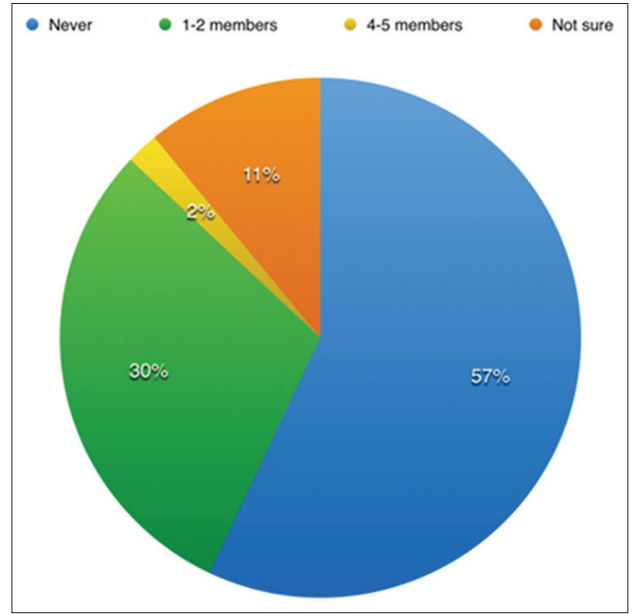

Fig. 2: Percentage of female sharing of borrowing makeup. Sample size-100

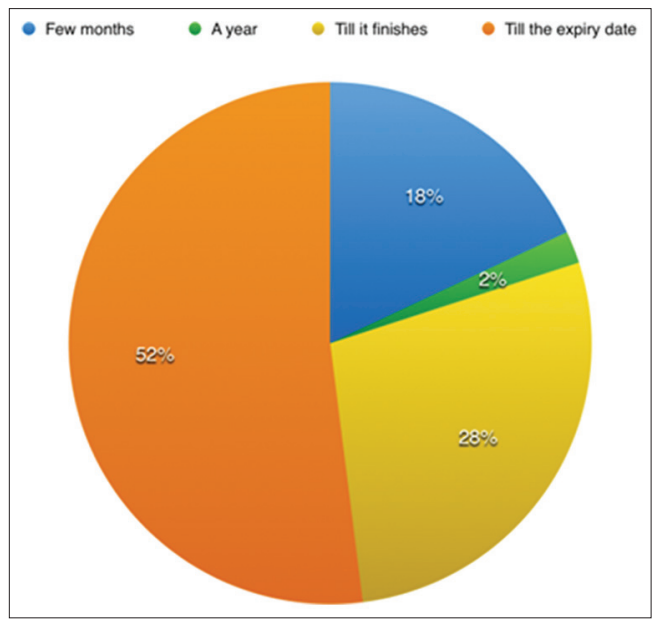

Fig. 3: Storage of makeup. Sample size-100

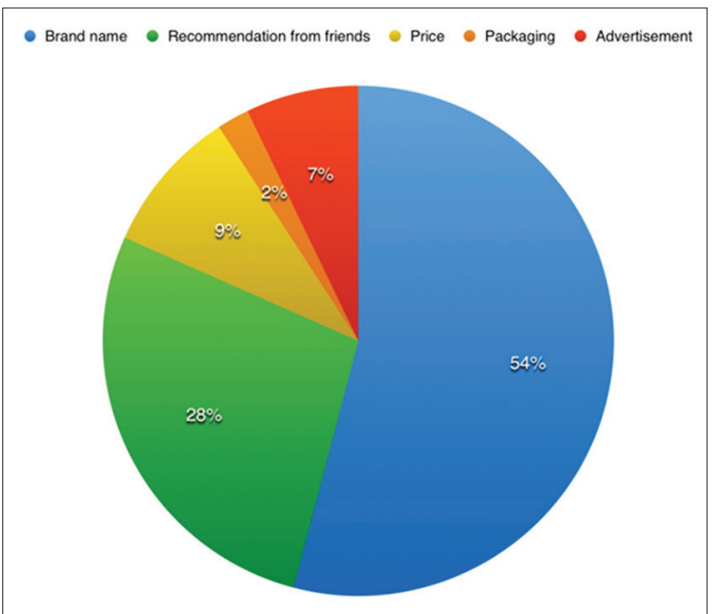

Fig. 4: Behavior of consumers toward cosmetic products. Sample size-100

the users prefer to avoid the use of cosmetic testers as it may become a major source for spread of infection. Approximately, $52 \%$ of the females who participated in the survey said that they take checked the products for ingredients before buying the products. In some products, it was found that the ingredients were not listed on the product, but on the box that it was sold in that is often discarded by the consumer. This is possibly one of the reasons why the rest did not check for ingredients in the product.

\section{DISCUSSION}

Cosmetics, in today's world, have become a part and parcel of every women's life. Unfortunately, the majority of the women are unaware of the harmful effects of the cosmetic products if not used properly. This research was conducted to determine the cosmetic contamination awareness among adolescent females. From the data analysis, it is clearly evident that the awareness regarding cosmetic usage and cosmetic contamination levels are on increase. Out of the 100 adolescent females who participated in the study, $87 \%$ of females use cosmetic products. It was determined that the probability of infection was low since most of the respondents used it correctly.

In general, the females who were surveyed showed that they knew the proper methods of applying as well as the duration of storage of makeup. A majority of the females participated in the survey prefer the use of bristle brushes over fingers, in which the latter could lead to an increase in chance of infection. Moreover, most (52\%) of them have also responded that they use makeup only until the expiry date which signifies great awareness among the adolescent females.

One of the major reasons for the outbreak of infection is the practice of sharing or borrowing makeup. From the data, it is visible that $57 \%$ of the females have responded that they never share makeup, which is a positive sign as sharing makeup increases the chances of microbial growth and infection for all users. Using cosmetic testers available in public stores is even worse because it is impossible to figure out who used it before you and whether they had an infection or not [4]. From previous studies conducted by Dawson, 1982, it is clearly evident that the unrestricted use of cosmetic testers, for which there are no sterile applicators, can spread bacteria from one person to another and possibly cause eye infections. A majority of the people who participated in the survey seems to have avoided the usage of cosmetic testers.

Brand name has a major influence on the buying behavior of the females. According to studies conducted by Okeke, 2001, contamination rates of cosmetics in North America and Europe are $2-43 \%$ and considering that undeveloped countries may not have strict regulations for manufacturers, it is important to be aware of foreign cosmetic brands and imports.

When it comes to awareness regarding the presence of toxic substances like lead and carcinogens and some preservatives which would harbor bacterial growth after a period of time, $87 \%$ of them have responded with a yes to the question. These responses from the adolescent females who completed the survey are a positive sign toward the rapid increase in the awareness of cosmetic contamination among the younger generation.

\section{CONCLUSION}

Each day women smear anti-aging, anti-blemish, cover-up, foundation, powder and more on their faces in the name of beauty. Even though we do not ingest it, skin is the largest organ in our body and absorbs chemicals directly into the bloodstream faster than if it were digested. The digestive system has a natural process to filter toxins, whereas the epidermis acts more like a sponge [12]. Thus, there is a great need to take into consideration the ingredients which go into the making of the products. The results of the study show the increase in cosmetic usage patterns and cosmetic contamination awareness among the adolescent females in the past few decades. 


\section{REFERENCES}

1. Schneider G, Gohla S, Schreiber J, Kaden W, Schönrock U, SchmidtLewerkühne H, et al. "Skin Cosmetics" in Ullmann's Encyclopedia of Industrial Chemistry. Weinheim: Wiley-VCH; 2005.

2. Nigam P K. Adverse reactions to cosmetics and methods of testing. Indian J Dermatol Venereol Leprol 2009;75(1):10-9.

3. Persaud L. Cosmetic Contamination Awareness Among Adolescent Females. Brooklyn, NY: Brooklyn Technical High School; 2007.

4. Anonymous. U.S. Food and Drug Administration. USA, Silver Spring, MD: FDA; 2011.

5. Haftbaradaran B, Abedi D, Jalali M, Bagherinejad MZ. Microbial quality survey of sunscreen products in Iranian market. Adv Biomed Res 2014;3:180.
6. Dawson RM. Data for Biochemical Research (Rex Malconten Chaplin). 2nd ed. Oxford, Oxfordshire: Oxford University Press; 1982.

7. Brannan DK, Dille JC. Types of closure prevents microbial contamination of cosmetics during consumer use. Appl Environ Microbiol 1990;56(5):1476-9.

8. Perry B. Cosmetic microbiology. Microbiol Today 2001;28(11):185-7.

9. Bhadauria R, Ahearn DG. Loss of effectiveness of preservative systems of mascaras with age. Appl Environ Microbiol 1980;39(3):665-7.

10. Hugbo PG. Onyekweli AO, Igwe I. Microbial contamination and preservative capacity of some brands of cosmetic creams. Trop J Pharm Res2003;2(2):299-34.

11. Lisbeth PM Toxins in Cosmetics - Contaminants in Your Personal Care Products. Available from: http://www.hormonesmatter.com/toxinscosmetics/. 